\title{
Kamcili-Yildiz, Naciye (202 I). Zwischen Glaubensvermittlung und Reflexivität. Eine quantitative Studie zu professionellen Kompetenzen von islamischen ReligionslehrerInnen (Internationale Hochschulschriften 682). Münster: Waxmann. ISBN 978-3-8309-4295-5. 276 Seiten.
}

\author{
Julia Bubenheim \\ Technische Universität Dortmund (julia.bubenheim@tu-dortmund.de)
}

Naciye Kamcili-Yildiz erhebt in ihrer quantitativ-empirischen Studie, die 2020 an der Universität Duisburg-Essen als Dissertation angenommen wurde, Einstellungen, Haltungen, fachwissenschaftliche und fachdidaktische Kompetenzen von muslimischen Religionslehrerinnen und -lehrern. Damit schließt dieses empirische Projekt eine Forschungslücke, indem es „erstmalig“ (13) professionelles Handeln und Kompetenzen muslimischer Religionslehrerinnen und -lehrer „mit Methoden der empirischen Bildungsforschung“ (205) untersucht. Als muslimische Religionslehrerin und Religionspädagogin an der Universität Paderborn ist es Naciye Kamcili-Yildiz möglich, eigene Erfahrungen und Erkenntnisse mit muslimischen Religionslehrerinnen und -lehrern sowie muslimischen Lehramtsanwärterinnen und -anwärtern zu reflektieren.

Sowohl der Buchtitel als auch das umfangreiche Inhaltsverzeichnis geben einen ersten Einblick und wecken die Leselust. Der Buchtitel ist gut gewählt, da er die empirische Fragestellung bereits offenlegt und hervorhebt, dass Glaubensvermittlung und Reflexivität wichtige professionelle Kompetenzen von muslimischen Religionslehrerinnen und -lehrern sind. Ein Aufgreifen und Ausführen des Buchtitels, beispielsweise in Kapitel 1 „Einleitung“ oder in Kapitel 9 „Fazit“ wäre m. E. nach hilfreich gewesen, um zu verdeutlichen, ob es sich bei der "Glaubensvermittlung“ und „Reflexivität“ um eine doppelte Zielrichtung, zwei wichtige Schwerpunkte oder zwei entgegengesetzte Pole der professionellen Kompetenzen von muslimischen Religionslehrerinnen und -lehrern handelt. Das Inhaltsverzeichnis sowie strukturleitende Meta-Kommentare und -Begründungen leisten eine angemessene Orientierung und Lenkung, sodass den Leserinnen und Lesern stets transparent vor Augen geführt wird, welche Leseabschnitte wann und warum anschließen.

In Kapitel 2, das die „Situation und Strömungen der islamischen Religionspädagogik“ behandelt, lassen sich insgesamt spannende Gegenwartsanalysen finden, die durch Schulbuchseiten, Tabellen und Grafiken konkretisiert und veranschaulicht werden. Auch die Tabellen und Grafiken in Kapitel 6 und 7 tragen zum Verständnis der komplexen Erhebungsinstrumente bei. Immer wieder hebt Naciye KamciliYildiz die Zentralität des Korans für Lernprozesse hervor. Sie stellt fest, dass der Koran bislang noch nicht ausreichend inhaltlich im islamischen Religionsunterricht erschlossen wird und eröffnet unter 2.7.1 einen möglichen Zugang mit dem Kinderkoran als Medium. Die breite Präsentation an religionspädagogischen Ansätzen und Projekten (Kapitel 2.7.3.3-2.7.3.8) scheint m. E. nicht ganz in der empirischen Untersuchung und ihrer Auswertung eingeholt zu sein, was Naciye Kamcili-Yildiz auch selbstkritisch in Kapitel 8.3 "Kritischer Rückblick und Ausblick“ reflektiert und als Desiderat für künftige Forschungsprojekte benennt (229). Infolgedessen kommt es zu einem leichten Bruch des Leseflusses zwischen Kapitel 2 und 3, da das Kapitel 2.6 „Zusammenfassung der Ergebnisse“ weniger eine Zusammenfassung ist, stattdessen eher ein spannender Exkurs über die historische Entwicklung der Moschee als Lernort.

Der theoretische „Vorbau“ (Kapitel 1-6) mit Kapitel 3 „Das Schulfach islamischer Religionsunterricht 
in Nordrhein-Westfalen", der pointierten Zusammenfassung der Studien COACTIV und FALKO-R, an die Naciye Kamcili-Yildizs Studie anschließt (Kapitel 4), die Definition des Kompetenzbegriffes (Kapitel 5) sowie Ziele und Anlagen der Untersuchung (Kapitel 6) sind sehr ausführlich ausgearbeitet. Naciye Kamcili-Yildiz setzt ihre Fragestellung und Ergebnisse (Kapitel 6-7) immer wieder mit Erkenntnissen der empirischen Bildungsforschung in Beziehung und zieht häufige Vergleiche zur katholischen und evangelischen Religionspädagogik, sodass ihre Forschung im interreligiösen und interkonfessionellen Fachdiskurs sehr gut verortet ist. Auch für nicht-muslimische Leserinnen und Leser, beispielsweise für christliche Religionslehrerinnen und -lehrer, sind ihre Ausführungen verständlich und nachvollziehbar. Die Testgütekriterien sind erfüllt und transparent dargestellt (Kapitel 7.2). Der standardisierte Fragebogen generiert interessante Erkenntnisse und eröffnet viele Möglichkeiten für Folgestudien und weitere Forschungsdesiderate, die Naciye Kamcili-Yildiz konsequent benennt (195-186, 228-231, 251-252 u. v. m.). Sie stellt fest, dass eine intrinsische anstelle einer extrinsischen Motivation bei muslimischen Religionslehrerinnen und -lehrern überwiegt. Muslimische Religionslehrerinnen und -lehrer überschätzen sich nach ihrer Untersuchung im Bereich von fachwissenschaftlichen und fachdidaktischen Kompetenzen meist selbst. Außerdem stellt Naciye Kamcili-Yildiz fest, dass eine hohe fachwissenschaftliche Kompetenz „keine hinreichende Bedingung [...] für fachdidaktisches Wissen ist" (209). Sie zeichnet immer wieder die eindeutige Verwobenheit von kulturell bedingten Traditionen und Religion heraus und die damit verbundene innermuslimische Heterogenität (189, 192, 224 u. v. m.).

Ihre spannenden empirischen Erkenntnisse (zusammengefasst in Kapitel 8.1-8.3) münden in einen Exkurs über das religionspädagogische Habitus-Modell (Kapitel 8.4). Dies hätte m. E. anhand von Konkretisierungen noch ertragreicher sein können. Naciye Kamcili-Yildiz hebt beispielsweise fortwährend das Potenzial einer Weiterentwicklung der Zertifikatskurse durch wiederkehrende Fortbildungsangebote hervor: Das religionspädagogische Habitus-Modell „eignet sich bei Fortbildungsveranstaltungen als Grundlage, um Bereiche zu identifizieren, in denen die islamischen Religionslehrkräfte noch Unterstützung brauchen“ (238-239). Hier wären Beispiele und Ausführungen hilfreich, damit das religionspädagogische Habitus-Modell nicht "nur" wie ein Exkurs wirkt, sondern integraler und bedeutender Bestandteil ihrer Forschung ist.

Insgesamt lässt sich sagen, dass die Monografie von Naciye Kamcili-Yildiz eine Bereicherung für die Forschungslandschaft darstellt und eine Forschungslücke im Bereich der empirischen Bildungsforschung von muslimische Religionslehrinnen und -lehren schließt. Naciye Kamcili-Yildiz liefert spannende Erkenntnisse, die in der universitären Lehramtsausbildung, in den Zertifikatskursen und im Kontext von Fortbildungen produktiv aufgegriffen werden können. Dabei verortet sie sich angemessen im Fachdiskurs der empirischen Bildungsforschung und im interreligiösen sowie interkonfessionellen Fachaustausch der christlichen und islamischen Religionspädagogik. Naciye Kamcili-Yildizs Forschung eröffnet viele weitere interessante Forschungsfragen. Dementsprechend bleibt zu hoffen, dass ihre Veröffentlichung den Ausgangspunkt für eine Reihe weiterer Publikationen bildet, um die gewonnenen Einsichten ihrer Forschung weiter zu vertiefen. 